\title{
Analysis of the equivalent indenter concept used to extract Young's modulus from a nano-indentation test: some new insights into the Oliver-Pharr method
}

\author{
Andriollo, Tito; Thorborg, Jesper; Hattel, Jesper Henri
}

Published in:

Modelling and Simulation in Materials Science and Engineering

Link to article, DOI:

$10.1088 / 1361-651 X / a a 6831$

Publication date:

2017

Document Version

Peer reviewed version

Link back to DTU Orbit

Citation (APA):

Andriollo, T., Thorborg, J., \& Hattel, J. H. (2017). Analysis of the equivalent indenter concept used to extract Young's modulus from a nano-indentation test: some new insights into the Oliver-Pharr method. Modelling and Simulation in Materials Science and Engineering, 25(4), [045004]. https://doi.org/10.1088/1361-651X/aa6831

\section{General rights}

Copyright and moral rights for the publications made accessible in the public portal are retained by the authors and/or other copyright owners and it is a condition of accessing publications that users recognise and abide by the legal requirements associated with these rights.

- Users may download and print one copy of any publication from the public portal for the purpose of private study or research.

- You may not further distribute the material or use it for any profit-making activity or commercial gain

- You may freely distribute the URL identifying the publication in the public portal 


\title{
Analysis of the equivalent indenter concept used to extract Young's modulus from a nano-indentation test: Some new insights into the Oliver- Pharr method.
}

Tito Andriollo ${ }^{1,3}$, Jesper Thorborg ${ }^{1,2,4}$ and Jesper Hattel ${ }^{1,5}$

${ }^{1}$ Department of Mechanical Engineering, Technical University of Denmark, DK-2800 Kgs. Lyngby, Denmark

${ }^{2}$ MAGMA GmbH, D-52072 Aachen, Germany

${ }^{3}$ Corresponding author. Address: Technical University of Denmark, Produktionstorvet, Building 425, room 225, 2800 Kgs. Lyngby, Denmark. Phone: +45452547 22. E-mail: titoan@mek.dtu.dk

${ }^{4}$ Address: Technical University of Denmark, Produktionstorvet, Building 425, room 232, 2800 Kgs. Lyngby, Denmark. E-mail: jest@mek.dtu.dk

${ }^{5}$ Address: Technical University of Denmark, Produktionstorvet, Building 425, room 228, 2800 Kgs. Lyngby, Denmark. E-mail: jhat@mek.dtu.dk

\begin{abstract}
In this paper a thorough analysis of the equivalent indenter concept applied to nano-indentation is carried out, motivated by the fact that previous works in the field have not considered the requirement of a consistent relation between contact depth and projected contact area. Dimensional analysis is initially used to prove that the shape of the axisymmetric equivalent indenter can be regarded as a material property, provided that size-effects are negligible. Subsequently, it is shown that such shape can effectively be employed to describe the nanoindentation unloading stage by means of Sneddon's elastic solution which is formally valid only for indentation into a flat surface. This allows for formulating the problem of extracting Young's modulus from the unloading curve as an optimization problem. However, it is proved that the latter does not have a unique solution, due to the particular mathematical structure of the underlying equations; hence, additional constraints are needed to set restrictions on the admissible equivalent indenter shapes. An example of such constraint is hidden in some apparent inconsistencies of the well-known Oliver-Pharr method, which is demonstrated to be based on an equivalent conical indenter whose semi-apical angle depends on the ratio between residual and total penetration. Specifically, this angle tends to 90 degrees when the material exhibits extensive inelastic deformation, whereas it reduces to the one characteristic of the real indenter for a perfectly elastic material. This provides a new physical explanation for the relatively good accuracy of the method even in presence of a non-negligible residual contact impression on the sample.
\end{abstract}




\section{Keywords}

Indentation; Analytical solutions; Young's modulus; Elastic modulus; Oliver-Pharr method; Equivalent indenter

\section{Article structure}

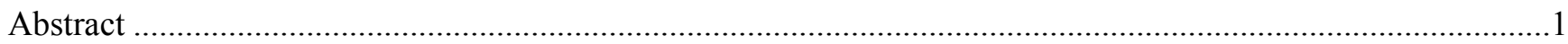

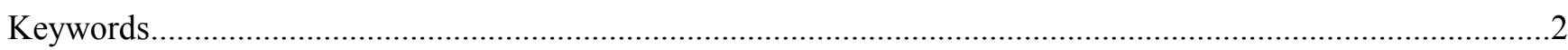

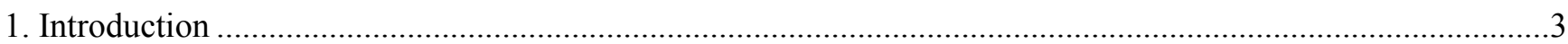

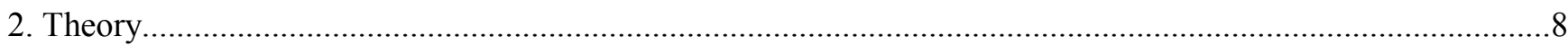

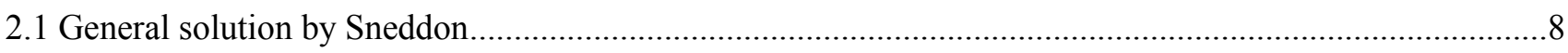

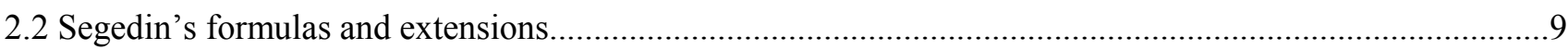

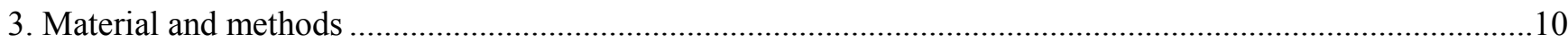

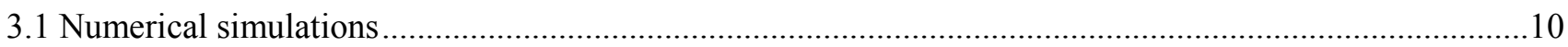

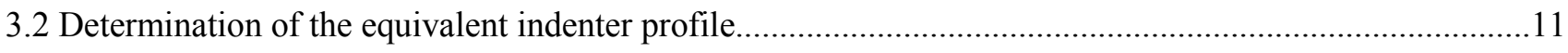

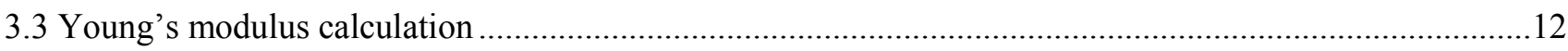

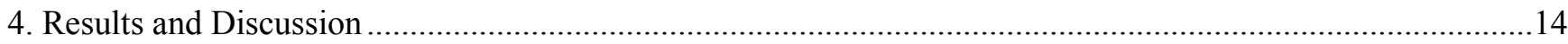

4.1 Functional dependence of the equivalent indenter shape ...................................................................14

4.2 Shape of the equivalent indenter profile from finite element simulations.............................................15

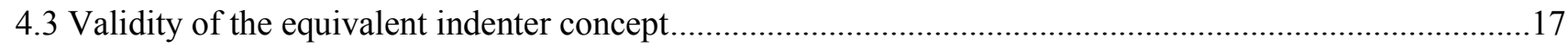

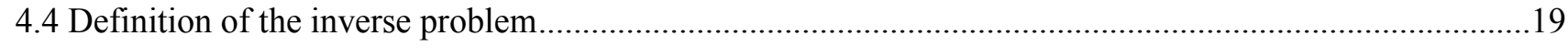

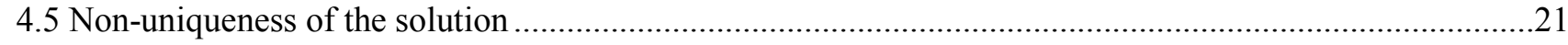

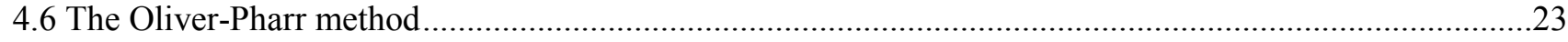

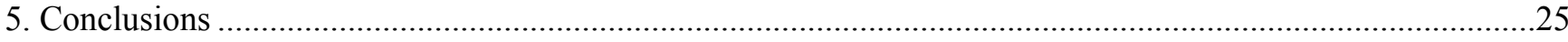

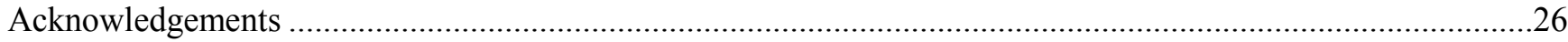

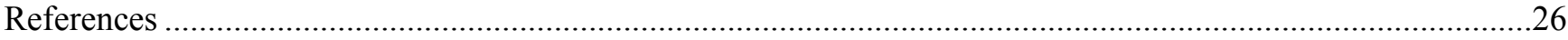




\section{Introduction}

Instrumented nano-indentation is a well-established technique which is widely used for probing mechanical properties of materials at length scales in the sub-micrometer range. Its high scientific relevance combined with a relatively simple experimental setup have attracted the attention of many researchers in the last decades, and several dedicated review papers exist which offer extensive accounts of the subject, e.g. (Fischer-Cripps, 2006)(Oliver and Pharr, 2004)(Cheng and Cheng, 2004).

In essence, a nano-indentation test consists in pressing a 3-sided pyramidal Berkovich indenter onto the surface of the sample under investigation and recording the resulting applied force vs penetration curve (figure 1-a). The main difference compared to traditional indentation techniques performed at the micron or millimeter scale is that the area of the residual impression left on the material is normally not measured, due to its much reduced size. For this reason, mechanical properties are usually estimated on the basis of the loading and unloading characteristics alone. Concerning this, a number of methods have been proposed so far which allow deriving parameters related to elastic and plastic material behavior, fracture toughness, creep, impact resistance, etc., a full list of which has recently been given in a comprehensive monograph (Fischer-Cripps, 2011). Focusing in particular on the determination of the isotropic elastic constants from nano-indentation data, the methodology initially proposed by (Doerner and Nix, 1986) and later extended and improved by (Oliver and Pharr, 1992) is

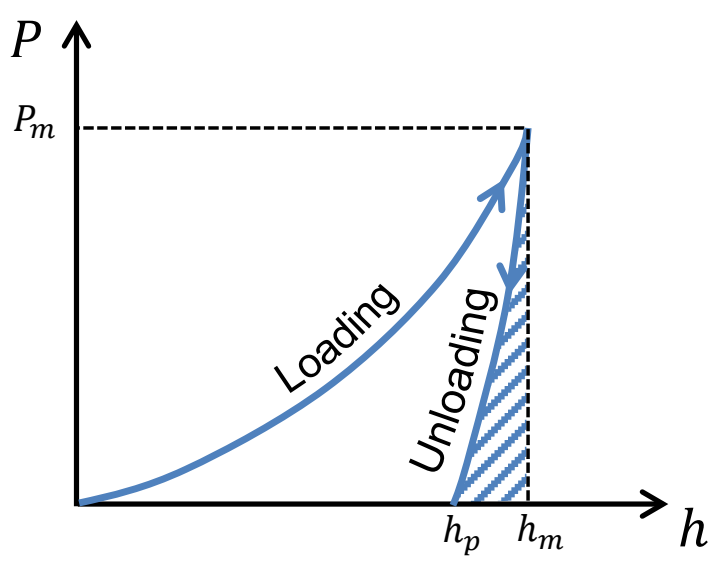

(a)

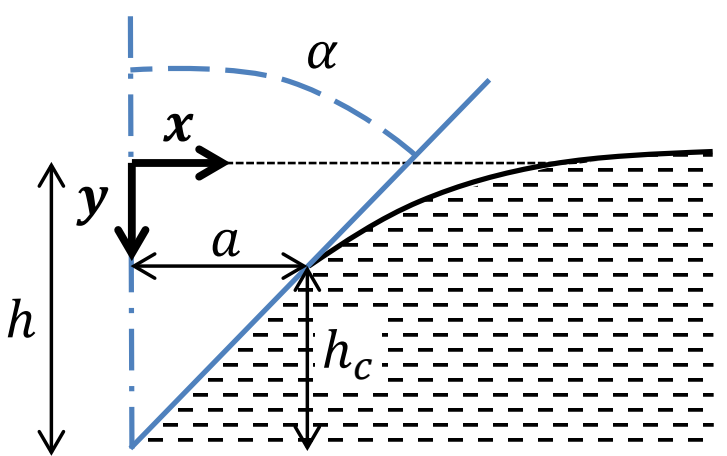

(b)

Figure 1: Nano-indentation test. (a) Recorded load vs penetration curve. The hatched area indicates the elastic work associated with the unloading stage. (b) Definition of the main geometrical quantities for axisymmetric conical indentation: $\alpha$ is the indenter semi-apical angle, $h$ is the total indenter penetration, $h_{c}$ is the contact depth and $a$ is the contact radius. 
probably the most well-known. The technique relies on a closed-form solution to the so-called Boussinesq's problem of indentation into an elastic half-space (Boussinesq, 1885), and it has proved very successful mainly due to its simplicity combined with relatively good accuracy.

Over the years, several modifications and improvements to the original Oliver-Pharr method have been suggested. Firstly, with respect to the experimental setup, continuous stiffness measurements have been introduced to measure properties as a continuous function of the penetration depth (Pharr et al., 2009)(Hay et al., 2010). In addition to enabling a very precise detection of the point of initial contact between the indenter and the sample, these measurements provide the possibility to analyze the moduli of materials which show pronounced time dependent deformation. As a consequence, nano-indentation based characterization of polymers and soft biological tissues has gained considerable interest in recent times, as mentioned in the reviews by (Gibson, 2014) and (McKee et al., 2011). Secondly, methods to account for the influence of the substrate during indentation of thin films have been established. This issue, addressed in an early paper by (Nix, 1989), becomes relevant when the maximum indentation depth is approximately $10 \%$ of the film thickness, or even less in some cases (Saha and Nix, 2002). Recent contributions to this field include the work of (Ni and Cheng, 2005), (Xu and Pharr, 2006), (Wang et al., 2010) and (Hay and Crawford, 2011), among others. Finally, in order to account for the residual contact stress and for errors in the contact area calculation, new techniques based on partial modifications of the equations behind the Oliver-Pharr method have been devised as well (Gong et al., 2004)(Troyon and Huang, 2005), even though they do not appear to offer indisputable advantages in terms of accuracy (Troyon and Huang, 2006).

In parallel, significant efforts have been made to find reliable ways to determine the elastic properties for materials which pile-up considerably, for which the Oliver-Pharr method is often inapplicable (Fischer-Cripps, 2006). To circumvent the problem of estimating the correct contact area between the sample and the indenter, which is the main cause of uncertainty in this case, (Cheng and Cheng, 1998) and (Cheng et al., 2002) proposed dimensionless relations connecting Young's modulus to experimentally accessible quantities. The same idea was the basis of the work of (Dao et al., 2001) and (Wang and Rokhlin, 2005), who constructed dimensionless functions from fitting the results of a large number of finite element simulations of the indentation process. Strategies to optimize the data-fitting procedure via algorithms based on neural networks were proposed later by (Tyulyukovskiy and Huber, 2006) and (Tyulyukovskiy and Huber, 2007). Unfortunately, an intrinsic drawback of this approach is the range of validity of the derived dimensionless relations, which are typically obtained under specific assumptions for the material inelastic behavior during loading. As a consequence, they should be used with caution for materials whose constitutive response is either completely unknown or remarkably different from the one employed in the numerical simulations. To overcome this limitation, methods which make use of additional experimental quantities, for instance measurements of the residual surface profile using the 
atomic force microscope ( $\mathrm{Li}$ et al., 2002)(Mata and Alcala, 2003) or of the contact area at maximum load using electrical resistance techniques (Poon et al., 2008a) have been devised as well. Despite delivering very precise results, the application of these procedures has been limited somehow by the much higher degree of experimental complexity they involve, which seems in contrast to the conceptual simplicity of a nanoindentation test. Therefore, it may be concluded that it does not seem possible to improve the accuracy of the Oliver-Pharr method without paying a price in terms of complexity and/or range of validity of the measurements. This is probably the reason why this method is still largely adopted in the scientific community, despite some of its aspects may appear difficult to understand at first glance.

Particularly, how purely elastic solutions developed for axisymmetric indenters (Love, 1939)(Harding and Sneddon, 1945)(Sneddon, 1948)(Segedin, 1957)(Sneddon, 1965) can effectively be used to analyze nanoindentation data, which is normally affected by an appreciable amount of inelastic deformation, is a matter which deserves a special comment. The very first point to note is that the Oliver-Pharr method considers only the unloading part of a nano-indentation test, which is assumed to be an entirely elastic process. Furthermore, the force-penetration curve produced by the pyramidal Berkovich indenter is supposed to be the same as that generated by a conical indenter with the same area-to-depth ratio. Extensive finite element calculations and experimental investigations have confirmed that these two assumptions are either fully satisfied, or lead to negligible errors in almost all practical cases (Dao et al., 2001)(Pharr and Bolshakov, 2002)(Poon et al., 2008a)(Sakharova et al., 2009).

Besides the points just discussed, another critical aspect exists which relates to the applicability of solutions obtained for indentation into a perfectly flat half-plane to the description of the unloading stage, which involves contact with a surface containing a residual impression. In principle, this particular contact condition can be described by the equivalent indenter concept (Bolshakov et al., 1995), whose profile is determined pointwise by the vertical distance between the real indenter and the profile of the sample surface after unloading, as schematically shown in figure 2 . That is, the elastic solutions previously mentioned can be effectively used to model the unloading stage provided that the equivalent indenter geometry is entered into the equations. 
According to (Woirgard and Dargenton, 1997)(Woirgard et al., 1998a)(Pharr and Bolshakov, 2002), the OliverPharr method is intrinsically based on the equivalent indenter concept. This justifies the relative good accuracy of the method, achieved even when the residual impression left on the sample is actually not negligible. In particular, the dependency on the equivalent geometry is reflected explicitly in the value of the parameter $\epsilon$ appearing in the formula

$$
h_{c}=h-\epsilon \frac{P}{P^{\prime}}
$$

which allows calculating the contact depth $h_{c}$ from knowledge of the applied load $P$, the total downward displacement $h$ of the indenter tip relative to the undeformed sample surface and the slope $P^{\prime}$ of the unloading curve. Figure 1-b provides the geometrical definition of these quantities.

It is the present authors' opinion, however, that there are two aspects of the Oliver-Pharr method which do not appear to be fully consistent with the equivalent indenter concept, at least apparently. The first one is the way the projected contact area $A$ (equal to $\pi a^{2}$ in figure 1-b) is estimated after $h_{c}$ has been calculated from equation (1). Indeed, an expression based on the real indenter geometry is assumed to hold, which usually has the form

$$
A=A_{r}\left(h_{c}\right)=C_{0} h_{c}^{2}+C_{1} h_{c}+C_{2} h_{c}^{1 / 2}+C_{3} h_{c}^{1 / 4}+\cdots
$$

where the coefficients $C_{1}, C_{2}, C_{3}, \ldots$ account for tip rounding effects and are normally obtained from calibration on fused silica samples (Oliver and Pharr, 2004). In the hypothetic situation of an indenter with ideal geometry, this expression reduces to

$$
A=A_{r}\left(h_{c}\right)=\pi \tan ^{2}(\alpha) h_{c}^{2}
$$

where $\alpha=70.3^{\circ}$ is the semi-apical angle of the conical punch with the same area-to-depth ratio of the

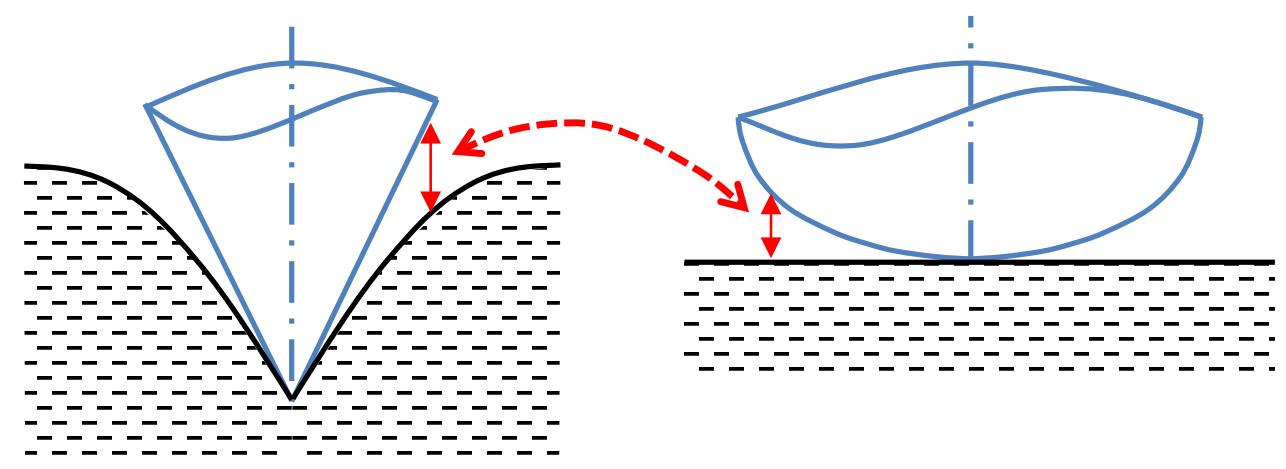

(a)

(b)

Figure 2: Definition of the equivalent indenter concept. (a) Real conical indenter after unloading. (b) Equivalent indenter geometry. 
Berkovich indenter. The key point is that, within the equivalent indenter framework, one would expect the contact area to be determined by means of the relation $A=A_{e q}\left(h_{c}\right)$ proper to the equivalent indenter instead. Clearly, it should be recognized that the relation $A=A_{e q}\left(h_{c}\right)$ is generally unknown, as can be realized easily from figure 2. In fact, it depends on the shape of the residual impression left on the sample, which in turn is determined by the mechanical properties of the material under investigation.

The second aspect of the Oliver-Pharr method which is seemingly in contrast to the equivalent indenter concept is the particular value of $h$ used in equation (1). In fact, as previously said, $h$ is assumed to represent the total downward displacement of the indenter tip. At first glance, according to the geometrical construction of figure 2 it would appear more logical to assume $h$ to represent only the recoverable part of the penetration, i.e. the total minus the residual. In the standard Oliver-Pharr procedure, equation (1) is evaluated only at maximum load. Nevertheless, by assuming the equivalent indenter concept to hold, the elastic relations describing the unloading stage should be satisfied for any value of the load, and so should equation (1). However, it is easily seen that when the indenter separates from the sample at the end of the unloading stage, meaning that $h_{c}=0, h$ is equal to the residual penetration $h_{p}$ indicated in figure 1-a. But then, to satisfy the equation, some load $P \neq 0$ should still be transmitted across the contact surface, which is non-physical.

For the sake of clarity, it is useful to say at this point that with a value for the projected contact area at hand, the reduced Young's modulus $E^{*}$ can be found via the well-known relation between $A, E^{*}$ and $P^{\prime}$ as

$$
E^{*}=\frac{\sqrt{\pi}}{2 \beta} \frac{P^{\prime}}{\sqrt{\mathrm{A}}}
$$

where $\beta$ is a correction factor which normally takes the value of $\approx 1.05$ (Oliver and Pharr, 2004). Subsequently, the standard Young's modulus $E$ can be eventually calculated on the basis of the relation

$$
E^{*}=\frac{E}{1-v^{2}}
$$

assuming that the Poisson's ratio $v$ of the material being indented is known.

In light of the arguments put forth so far, it appears that the Oliver-Pharr method does not seem to be fully consistent with the equivalent indenter concept. This raises two questions. First of all, what would happen if a fully consistent method were used? Would it provide better results? Secondly, how to explain the relatively good accuracy of the Oliver-Pharr method then, since it does not satisfy the equivalent indenter assumptions? In other words, why does this method work anyway?

In order to clarify these points, it is the aim of the present paper to re-examine the application of the equivalent indenter concept to the Young's modulus estimation from nano-indentation unloading curves by means of classic analytical solutions to the Bousinnesq' problem. Initially, the latter will be reviewed and re-formulated at the 
same time in a more convenient dimensionless form. Subsequently, the validity of the equivalent indenter concept for the description of the contact condition with a deformed surface will be tested, emphasizing the level of accuracy which can be possibly obtained. After that, the inverse problem (Dao et al., 2001) will be defined and critically analyzed within a rigorous and consistent framework. Finally, solution strategies will be discussed, eventually establishing a link with the traditional Oliver-Pharr method and showing that its apparent inconsistencies are in reality associated with a concealed, convenient choice of the equivalent indenter profile.

\section{Theory}

In this section, the most common solutions available in the literature for axisymmetric indentation into an isotropic elastic half-space are presented and reformulated for convenience in a dimensionless format. The main geometrical parameters considered are shown in figure 1-b.

\subsection{General solution by Sneddon}

Let us assume that the axisymmetric profile of a rigid indenter is given by a smooth monotonic function

$$
y=f(x) \Rightarrow y=f(a \tilde{x})
$$

where $a$ is the contact radius and $\tilde{x}=x / a$ is a normalized x-coordinate. According to (Sneddon, 1965), the load $P$ and penetration $h$ corresponding to a certain value of $a$ are given by

$$
h=\int_{0}^{1} \frac{f_{\tilde{x}}^{\prime}(a \tilde{x})}{\sqrt{1-\tilde{x}^{2}}} d \tilde{x}, \quad P=2 E^{*} a \int_{0}^{1} \frac{\tilde{x}^{2} f_{\tilde{x}}^{\prime}(a \tilde{x})}{\sqrt{1-\tilde{x}^{2}}} d \tilde{x}
$$

where the symbol $f_{\tilde{x}}^{\prime}$ indicates the derivative of $f$ with respect to $\tilde{x}$

$$
f_{\tilde{x}}^{\prime}=\frac{d f}{d \tilde{x}}=\frac{d f}{d x} \frac{d x}{d \tilde{x}}=f^{\prime} a
$$

and $E^{*}$ is the reduced Young's modulus defined in equation (5).

The normalization with respect to the contact radius is somewhat inconvenient, as this quantity is usually an unknown for the problem. Therefore, it is useful to introduce a normalization based on a length scale which is directly available from an indentation test, for instance the maximum penetration $h_{m}$, which will be indicated with the symbol $\left(^{-}\right)$. Accordingly, relations (7) take the form

$$
\bar{h}=\int_{0}^{\bar{a}} \frac{f^{\prime}\left(h_{m} \bar{x}\right)}{\sqrt{1-(\bar{x} / \bar{a})^{2}}} d \bar{x}, \quad P=2 E^{*} h_{m}^{2} \int_{0}^{\bar{a}} \frac{\bar{x}^{2} f^{\prime}\left(h_{m} \bar{x}\right)}{\sqrt{\bar{a}^{2}-\bar{x}^{2}}} d \bar{x}
$$

Note that as $f^{\prime}$ is dimensionless, with this formulation the integrals appearing in the last two equations turn out to be dimensionless as well. 


\subsection{Segedin's formulas and extensions}

Let us assume that the function $f$ in equation (6) is given by the following polynomial expression

$$
\bar{y}=\sum_{n=1}^{N} \bar{b}_{n} \bar{x}^{n}, \quad \bar{b}_{n}=b_{n} h_{m}^{n-1}
$$

By introducing the coefficients

$$
\bar{c}_{n}=\sqrt{\pi} \frac{\Gamma\left(\frac{n}{2}+1\right)}{\Gamma\left(\frac{n}{2}+\frac{1}{2}\right)} \bar{b}_{n}
$$

where $\Gamma$ denotes the gamma-function, it is possible to demonstrate that the relation between normalized penetration depth $\bar{h}$ and contact radius $\bar{a}$ can be written in closed-form as

$$
\bar{h}=\sum_{n=1}^{N} \bar{c}_{n} \bar{a}^{n}
$$

Similarly, the expression for the load $P$ becomes

$$
P=2 E^{*} h_{m}^{2} \sum_{n=1}^{N} \frac{n}{n+1} \bar{c}_{n} \bar{a}^{n+1}
$$

The last two equations are commonly attributed to Segedin (Segedin, 1957).

Expressions (12) and (13) allow for deriving other useful relations. The load derivative with respect to normalized penetration is given by

$$
P^{\prime}=\frac{d P}{d \bar{h}}=\frac{d P}{d \bar{a}}\left(\frac{d \bar{h}}{d \bar{a}}\right)^{-1}=2 E^{*} h_{m}^{2} \bar{a}
$$

It is important to point out that the last relation, which justifies equation (4), can be obtained directly from the general relations (7), as demonstrated in (Pharr et al., 1992).

Similarly, the elastic work $W$ done during indentation, corresponding to the hatched area in figure $1-\mathrm{a}$, can be calculated as

$$
W=\int_{0}^{h} P d h=\int_{0}^{\bar{a}} P \frac{d h}{d \bar{a}} d \bar{a}=2 E^{*} h_{m}^{3} \sum_{n, s=1}^{N} \frac{n s}{(s+n)(n+1)} \bar{c}_{n} \bar{c}_{s} \bar{a}^{n+s+1}
$$

If only one term is retained in the expansion (10), the following direct relation between load and penetration may be obtained by combining equations (12) and (13) together: 


$$
P=2 E^{*} h_{m}^{2} \frac{n}{(n+1) \bar{c}_{n}{ }^{1 / n}} \bar{h}^{1+1 / n}
$$

For a conical indenter $n=1$. Then, equation (11) reduces to

$$
\bar{c}_{1}=\sqrt{\pi} \frac{\Gamma\left(\frac{1}{2}+1\right)}{\Gamma\left(\frac{1}{2}+\frac{1}{2}\right)} \bar{b}_{1}=\sqrt{\pi} \frac{\sqrt{\pi} / 2}{1} \bar{b}_{1}=\frac{\pi}{2} \bar{b}_{1}
$$

By inserting the latter result with $\bar{b}_{1}=\cot (\alpha)$ into equation (16) one obtains

$$
P=E^{*} h_{m}^{2} \frac{2}{\pi \cot (\alpha)} \bar{h}^{2}
$$

which represents the traditional load-penetration formula for a conical indenter of semi-apical angle $\alpha$, originally derived by Love (Love, 1939).

\section{Material and methods}

\subsection{Numerical simulations}

It seems logical to test the validity of the equivalent indenter concept for indented surfaces with realistic geometries. To avoid direct experimental measurements, which would require a huge investment of time and resources, nano-indentation tests are simulated via the finite element software ABAQUS. In this way, a large number of residual impression profiles can be generated without resorting to atomic force microscope profilometry.

As already explained in the introduction, previous investigations have demonstrated that the Berkovich indentation process can be analyzed by means of the simplified axisymmetric geometry depicted in figure 3-a, where the semi-apical angle of the conical indenter is set to $\alpha=70.3^{\circ}$ (Oliver and Pharr, 2004). The latter is represented in ABAQUS by an analytic rigid surface, whereas the sample is assumed to be made of a generic metallic material whose behavior is considered to be isotropic elasto-plastic. A standard large-strain J2-flow plasticity formulation is adopted and the material parameters are chosen as follows: Young's modulus is set to $E=100 \mathrm{GPa}$ and the yield stress $\sigma_{y}$ is varied in the range $1 \times 10^{-4} E \div 2 \times 10^{-2} E$, which should be sufficiently wide to cover values for all common metals (Ashby et al., 2010). Concerning hardening, following (Larsson et al., 1996) and (Bolshakov and Pharr, 1998) an isotropic linear law is used, with three different values for the hardening modulus $K: 0.05 E, 0.01 E$ and $0.002 E$. Finally, as Poisson's ratio of almost all metals lies between 0.25 and 0.35 (Greaves et al., 2011), a fixed mean value of 0.3 is adopted, except for those simulations in which the dependence of the results on this specific parameter is investigated explicitly.

The interaction between the cone and the sample surface is realized via a finite sliding, surface-to-surface contact discretization with zero friction, as previous studies have reported a minor influence of this last 
parameter on the results (Larsson et al., 1996)(Sakharova et al., 2009). In addition, in all simulations a maximum indentation depth of 1 micron is used, which is sufficiently deep to ensure that indenter tip rounding effects, often observed during real nano-indentation tests, do not need to be taken into account (Oliver and Pharr, 2004). Accordingly, the height and radius of the cylindrical sample are both set to 100 microns following the criterion proposed by (Poon et al., 2008b), which guarantees fulfillment of the "infinite" half-space assumption, common to all analytical solutions presented in the previous section.

The adopted mesh, visible in figure 3-b, consists of approximately 2000 axisymmetric quadrilateral hybrid elements CAX4H. To avoid excessive distortion close to the indenter tip during loading, an Arbitrary Lagrangean-Eulerian (ALE) adaptive technique is employed (Dassault Systèmes Simulia Corp., 2013). The quality of the mesh is sufficient to ensure a precision of $1 \%$ on the slope of the recorded $P$ vs $h$ curves.

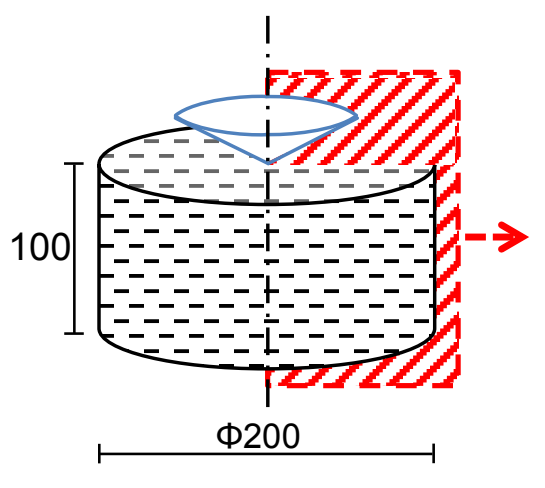

(a)
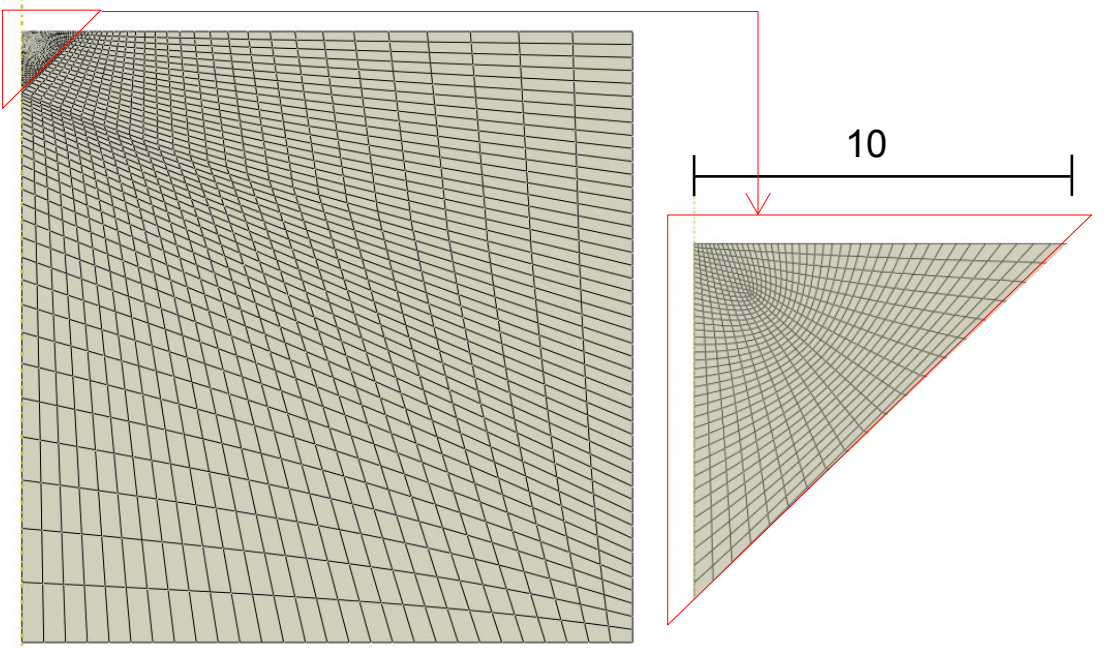

(b)

Figure 3: Finite element model for nano-indentation. (a) Geometry of the indenter and of the sample. (b) Mesh adopted. Dimensions are in microns.

\subsection{Determination of the equivalent indenter profile}

From each ABAQUS simulation, the shape of the residual impression after unloading is extracted in the form of a function $u_{r}(x)$, which describes the permanent downward displacement of the sample surface relative to the Cartesian coordinate system $(x, y)$ shown in figure 1-b.

The shape of the equivalent indenter profile turns out to be naturally defined by the function

$$
f_{e q}(x)=u_{r}(x)-\left(h_{p}-x \cot (\alpha)\right)
$$


where $h_{p}$ is the recorded residual plastic penetration, visible in figure 1-a, and $\alpha$ is the semi-apical angle of the conical indenter. For convenience, the last expression can be re-cast in dimensionless form as follows:

$$
\bar{f}_{e q}(\bar{x})=\bar{u}_{r}(\bar{x})-\left(\bar{h}_{p}-\bar{x} \cot (\alpha)\right)
$$

In order to obtain a representation for the function $\bar{f}_{e q}(\bar{x})$ which can easily be used in combination with the analytical solutions presented in section 2, the following polynomial approximation is introduced:

$$
\bar{f}_{e q}(\bar{x}) \approx \sum_{n=1}^{N} \bar{b}_{n} \bar{x}^{n}
$$

The optimal choice of the coefficients appearing at the right-hand-side of the last expression depends on the extension of the domain considered for $\bar{f}_{e q}(\bar{x})$. In the present work, two possible options are investigated:

- The "contact" domain, suggested by (Pharr and Bolshakov, 2002): $\bar{x} \in\left[0, \bar{a}_{m}\right]$, where $\bar{a}_{m}$ is the normalized value of the contact radius at maximum load.

- The "extended" domain: $\bar{x} \in[0,5]$, which is approximately double the size of the previous one.

Figure 4 shows an example of the function $\bar{u}_{r}(\bar{x})$ extracted from ABAQUS, with indication of the two different domains mentioned above.

\subsection{Young's modulus calculation}

The $\bar{b}_{n}$ coefficients determined according to equation (21) can be employed to find analytical estimates for the sample Young's modulus using the simulated force-penetration curves as starting point. In fact, the two functions $P(t)$ and $\bar{h}(t)$ recorded during the elastic unloading stage can be extracted from ABAQUS and used together with the $\bar{b}_{n}$ to solve the system of equations (11)-(12)-(13) for the function $E^{*}(t)$, where $t$ indicates the relative time-coordinate with respect to the entire unloading process. Subsequently, conversion to the standard Young's modulus $E(t)$ may be easily performed by means of equation (5), assuming that Poisson's ratio is known.

The function $E(t)$ determined in this way turns out to be defined over 100 points, which simply correspond to the ABAQUS increments the unloading step is subdivided into. An average value of $E(t)$ may then be calculated on the basis of the upper $50 \%$ of the unloading data. The lower $50 \%$ is discarded for three main reasons. Firstly, it is affected by an artificial lack of smoothness due to the fact that the number of mesh elements in contact with the indenter reduces quickly, eventually becoming zero at $100 \%$ unloading. Secondly, as will be explained in the next section, it is sometimes necessary to correct the equivalent indenter profile close to the indenter tip. While this does not sort any significant effect on the top part of the unloading curve, it might adversely affect the results when the contact area becomes very small. Thirdly, in this way the analysis becomes 
more similar to a real nano-indentation test, in which it is common practice to consider only the upper part of the unloading curve for the calculation of the Young's modulus.

For the sake of clarity, it is important to remark that only the elastic part of the indenter tip penetration is entered in the analytical expressions presented in section 2, i.e. the difference between the absolute penetration relative to the original undeformed sample surface and the residual plastic penetration $\bar{h}_{p}$. Unless stated otherwise, $\bar{h}(t)$ and $\bar{h}$ will always denote this elastic part of the indenter penetration throughout the rest of the analysis.

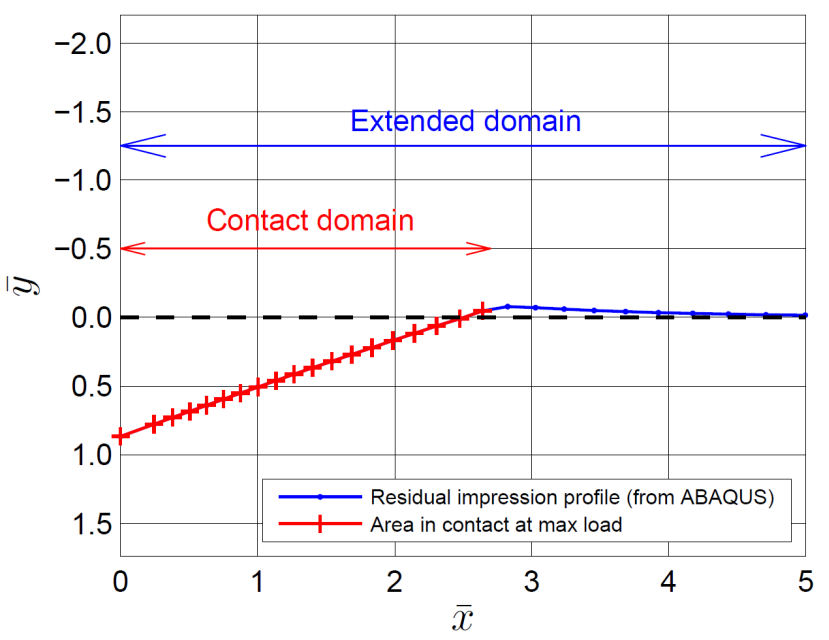

Figure 4: Example of residual impression profile extracted from ABAQUS, with indication of the two different domains considered to approximate the function $\bar{f}_{e q}(\bar{x})$. The data refer to a simulation run using $\sigma_{y} / E=K / E=1 \%$. 


\section{Results and Discussion}

\subsection{Functional dependence of the equivalent indenter shape}

Before attempting any description of the relation between force and penetration during unloading using the equivalent indenter concept, it is essential to examine whether or not the analytical form of the function $\bar{f}_{\text {eq }}(\bar{x})$ defined in (20) can be considered as a material property.

The first step to clarify this point is to write down explicitly the functional dependence of the function $u_{r}$ appearing in (19), which gives the shape of the residual impression. If size-effects are negligible, it is not difficult to realize that the variables influencing $u_{r}$ are the maximum penetration achieved during loading, the elastic and plastic material parameters and obviously the coordinate $x$ :

$$
u_{r}=u_{r}\left(h_{m}, E, v, \sigma_{y}, K, x\right)
$$

The dimension matrix associated with the latter expression has a rank equal to 2. As there are 7 physical quantities involved, Buckingham's Pi Theorem (Barenblatt, 1996) indicates that it can be rewritten in terms of $7-2=5$ dimensionless variables. For the sake of convenience, the following dimensionless groups are chosen:

$$
\bar{u}_{r}=\frac{u_{r}}{h_{m}}, \quad \bar{x}=\frac{x}{h_{m}}, \quad v, \quad \frac{\sigma_{y}}{E}, \quad \frac{K}{E}
$$

so that (22) becomes

$$
\bar{u}_{r}=\Pi_{1}\left(v, \sigma_{y} / E, K / E, \bar{x}\right)
$$

Similar arguments may be used to show that the normalized residual penetration $\bar{h}_{p}$ has to be function of the last three dimensionless groups appearing in (23) (Dao et al., 2001):

$$
\bar{h}_{p}=\Pi_{2}\left(v, \sigma_{y} / E, K / E\right)
$$

By inserting relations (24) and (25) into (20) one obtains

$$
\bar{f}_{e q}(\bar{x})=\Pi_{1}\left(v, \frac{\sigma_{y}}{E}, \frac{K}{E}, \bar{x}\right)-\Pi_{2}\left(v, \frac{\sigma_{y}}{E}, \frac{K}{E}\right)+\bar{x} \tan (\alpha)
$$

The latter relation proves that the shape of the equivalent indenter, i.e. the analytical form of the function $\bar{f}_{e q}(\bar{x})$, depends only on dimensionless combinations of the material parameters and it is hence unaffected by the particular choice of the maximum indentation depth. This guarantees that the results are insensitive to the arbitrary values of $h_{m}$ and $E$ employed in the present numerical simulations.

Another important observation can be made regarding the $\bar{b}_{n}$ coefficients defined in (21), which are used to approximate $\bar{f}_{e q}(\bar{x})$. From a mathematical point of view, they can be seen as provided by an operator which accepts $\bar{f}_{e q}(\bar{x})$ and its domain size as input. In the context of the present analysis, the latter is either fixed or 
defined by the upper limit $\bar{a}_{m}$ as explained in section 3.2. However, straightforward application of Buckingham's Pi Theorem can show that the normalized value $\bar{a}_{m}$ of the contact radius at maximum load is also uniquely determined in terms of material properties (Cheng and Cheng, 1999). As a consequence, the $\bar{b}_{n}$ coefficients turn out to be material parameters as well.

\subsection{Shape of the equivalent indenter profile from finite element simulations}

Figure 5 shows the equivalent indenter profiles calculated for selected values of the plastic material parameters according to the procedure described in section 3.2. As the "contact" domain assumption is considered, all curves are interrupted in correspondence to the point where $\bar{x}=\bar{a}_{m}$. As already pointed out by (Pharr and Bolshakov, 2002), it can be seen that the function $\bar{f}_{e q}(\bar{x})$ is not linear, and the deviation from linearity is larger for higher values of the $\sigma_{y} / E$ ratio. On the other hand, in contrast to the observations of the previous authors, the function $\bar{f}_{e q}(\bar{x})$ determined from the present simulations is not always monotonic. For large $\sigma_{y} / E$ values a small initial decrease is visible, where $\bar{f}_{e q}(\bar{x})$ becomes negative. The reason is that during the final part of the unloading stage, the area of the sample in contact with the indenter gradually shrinks to a point which does not coincide with the indenter tip. As this local lack of monotonicity in $\bar{f}_{e q}(\bar{x})$ near $\bar{x}=0$ would preclude the application of the general Sneddon's equations (9), the equivalent profiles featuring this characteristic are modified by "flattening" their tips as shown in figure 6-a. It must be emphasized that since the maximum negative value of $\bar{f}_{e q}(\bar{x})$ is always small in comparison to the equivalent indenter size, less than $1 \%$ of the contact radius at maximum load, this shape modification does not sort any significant effects on how the upper

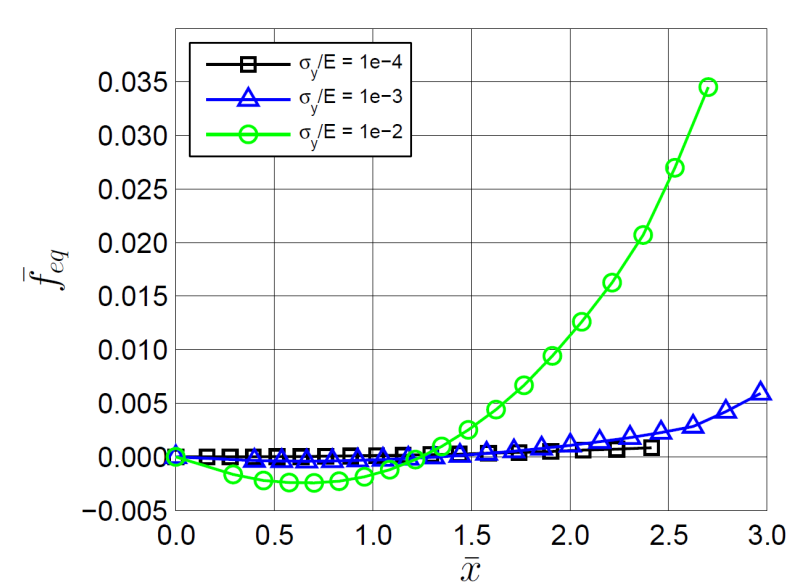

(a)

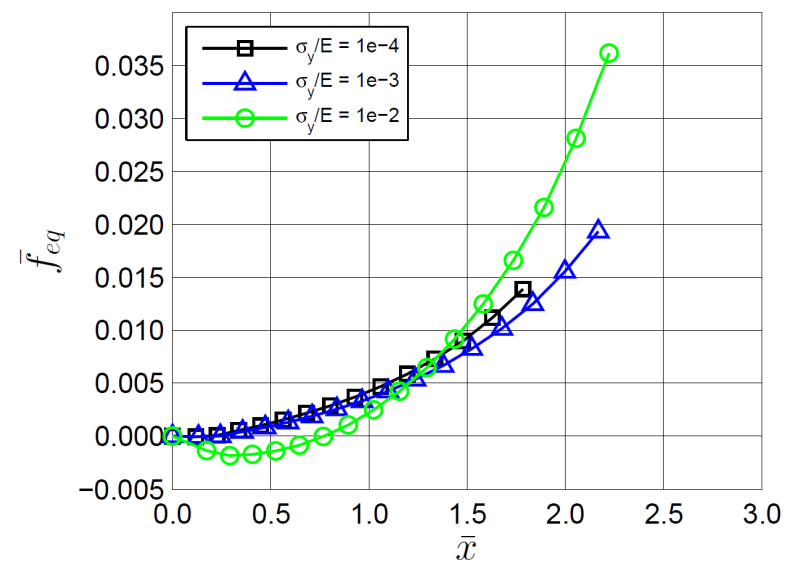

(b)

Figure 5: Calculated equivalent indenter profiles for selected values of the ratio $\sigma_{y} / E$, considering the "contact" domain. In (a) the ratio $K / E$ is $0.2 \%$, whereas in (b) it is $5.0 \%$. 

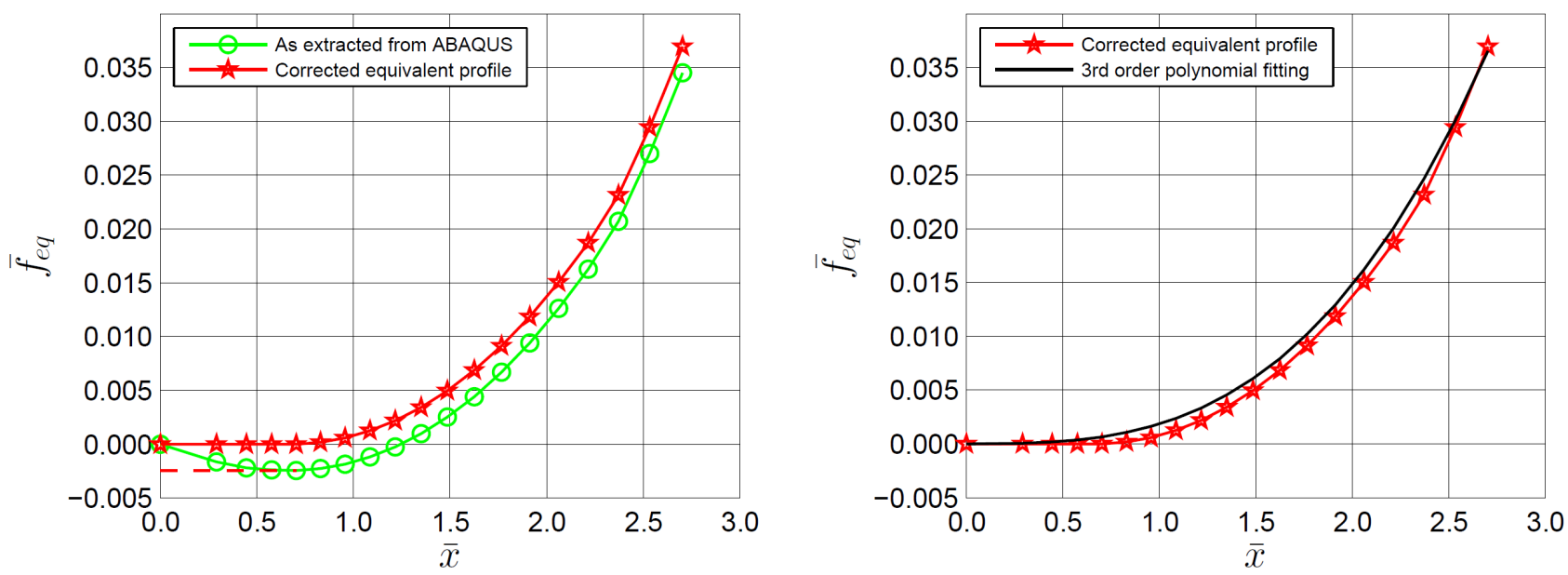

Figure 6: Analysis of the equivalent indenter profile corresponding to $\sigma_{y} / E=1 \%$ and $K / E=0.2 \%$. (a) Correction for local lack of monotonicity close to the indenter tip. (b) Third-order polynomial fitting of the equivalent indenter profile.

part of the unloading curve is described by the equivalent indenter.

The varying curvature of the profiles shown in figure 5 suggests seeking an approximation to $\bar{f}_{e q}(\bar{x})$ based on a $3^{\text {rd }}$ order polynomial. This means that only the first three terms are retained in equation (21). Moreover, preliminary analyses showed that the quadratic term did not play a major role, so that it can be neglected in order to keep the number of fitting parameters as low as possible. Consequently, the following expression is used to approximate $\bar{f}_{e q}(\bar{x})$ :

$$
\bar{f}_{e q}(\bar{x}) \approx \bar{b}_{1} \bar{x}+\bar{b}_{3} \bar{x}^{3}
$$

Figure 6-b shows an example of equivalent indenter profile fitted using the formula above. 


\subsection{Validity of the equivalent indenter concept}

On the basis of the $\bar{b}_{n}$ coefficients determined using equation (27), the procedure described in section 3.3 can be carried out to obtain estimates for the sample Young's modulus, which can then be compared with the reference value given as input to the finite element simulations. The agreement between the two provides a quantitative indication of the capability of the equivalent indenter geometry to describe the contact condition with a surface containing a residual impression.

Results obtained in this way are reported in figure 7-a. It can be noticed that a consistent overestimation of the Young's modulus (calculated on the basis of the upper $50 \%$ of the unloading data) seems to be present. Nevertheless, the maximum relative deviations from the reference value are limited to $10 \div 15 \%$ for all combinations of material parameters tested. In addition, the associated standard deviation, shown in figure 7-b, is always below $1.5 \%$. This is important, as in principle the same value of Young's modulus should be obtained independently of the particular point along the force-penetration curve considered for the calculation, i.e. the particular pair of $(P, h)$ values.

The consistent Young's modulus overestimation can be explained, at least partly, by the fact that all analytical solutions presented previously neglect the radial displacement of the material points in contact with the axisymmetric indenter. This issue was thoroughly addressed in the work of (Hay et al., 1999), where it was shown that Sneddon's equations are formally correct only for Poisson's ratio equal to 0.5 . Conversely, when a

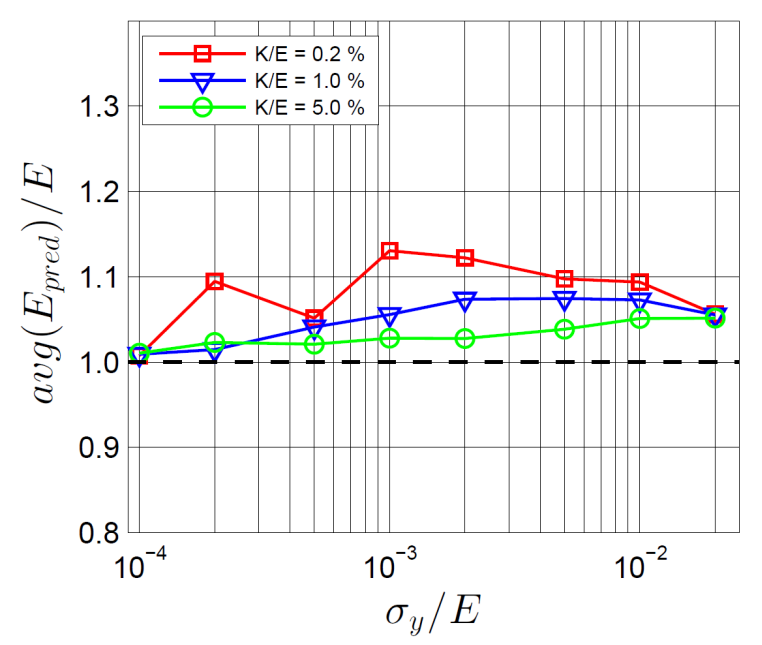

(a)

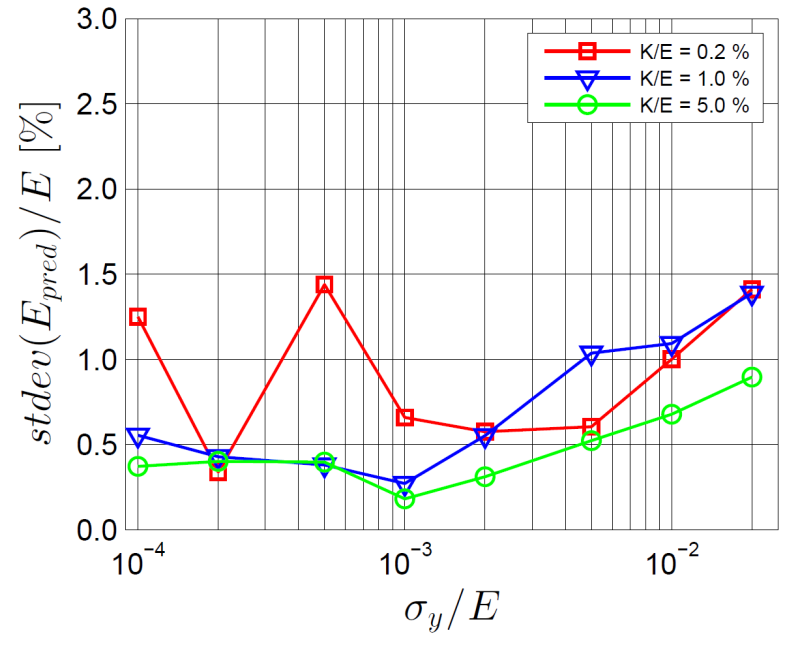

(b)

Figure 7: Results for third-order polynomial fitting of the equivalent indenter profile over the "contact" domain. (a) Mean value of the Young's modulus and (b) associated standard deviation. 
certain amount of material compressibility is introduced, a progressive error builds up, which leads to a Young's modulus overestimation of about $8 \%$ for a Poisson's ratio of 0.25 . The relevance of this aspect for the present analysis is made clear in figure 8 , which shows results obtained for different values of the latter parameter. It can be noticed that the deviation from the reference value of Young's modulus decreases as $v$ tends to 0.5 , in agreement with the considerations of the former authors. As a consequence, by implementing correction factors in the analytical solutions which take into account the influence of Poisson's ratio, such as the ones proposed in (Hay et al., 1999), the $10 \div 15 \%$ error bound could probably be reduced by a considerable amount. This means that the equivalent indenter concept is actually very suitable to model the particular contact problem arising during the unloading part of a nano-indentation test, provided that a sufficiently good approximation to the function $\bar{f}_{e q}(\bar{x})$ is available.

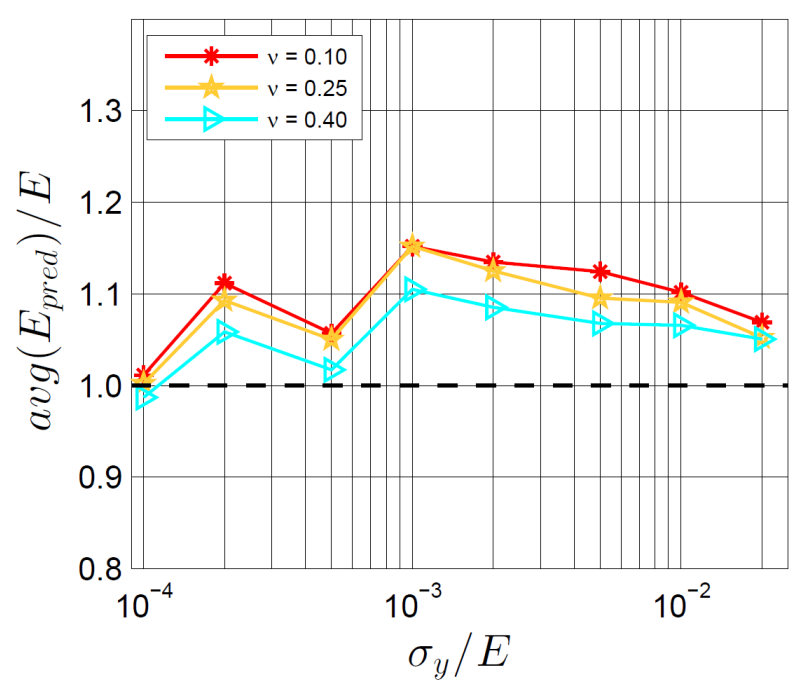

Figure 8: Effect of the Poisson's ratio on the calculated mean value of the Young's modulus using third-order polynomial fitting over the "contact" domain. A value of $K / E=0.2 \%$ is assumed.

Regarding this last point, previous works in the field have adopted almost exclusively power-law approximations to the equivalent indenter profile, of the type $\bar{f}_{e q}(\bar{x})=\bar{b}_{n} \bar{x}^{n}$ (Pharr and Bolshakov, 2002; Woirgard and Dargenton, 1997). The main advantage compared to a polynomial approximation is that the elastic analytical relation between load and penetration assumes the simple form of equation (16), implying that the following connection should exist between the exponent $n$, which describes the shape of the equivalent indenter, and the exponent $m$, which describes the power-law relation between load and penetration:

$$
m=1+1 / n
$$


The results reported in figure 9-a, based on the present simulations, indicate that this relation is to some extent satisfied, even though deviations as large as $50 \%$ are seen for some combinations of the material parameters. In addition, figure 9-b suggests that the accuracy of the power-law approximation is lower compared to the one associated with expression (27), despite both mathematical formulations possess the same number of fitting parameters. Therefore, low-order polynomials seem to be a better option for describing the shape of the equivalent indenter.

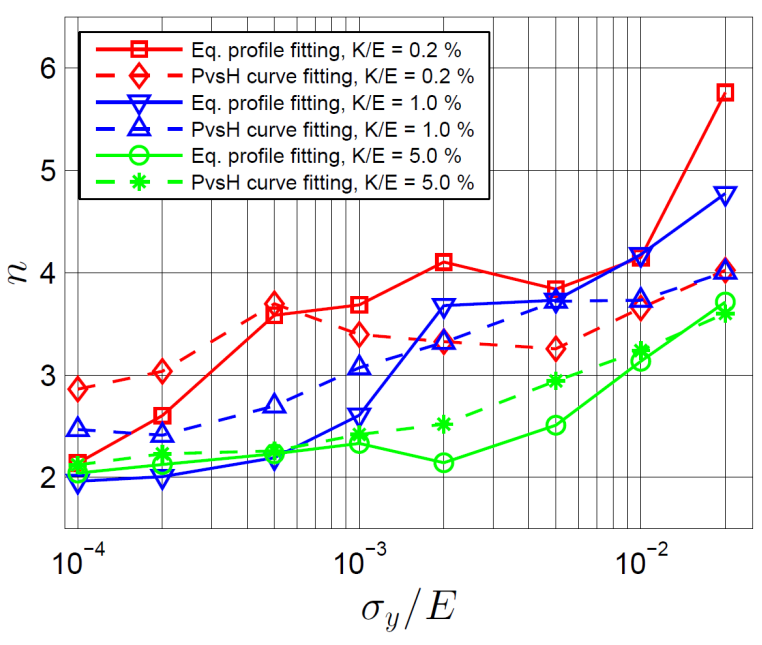

(a)

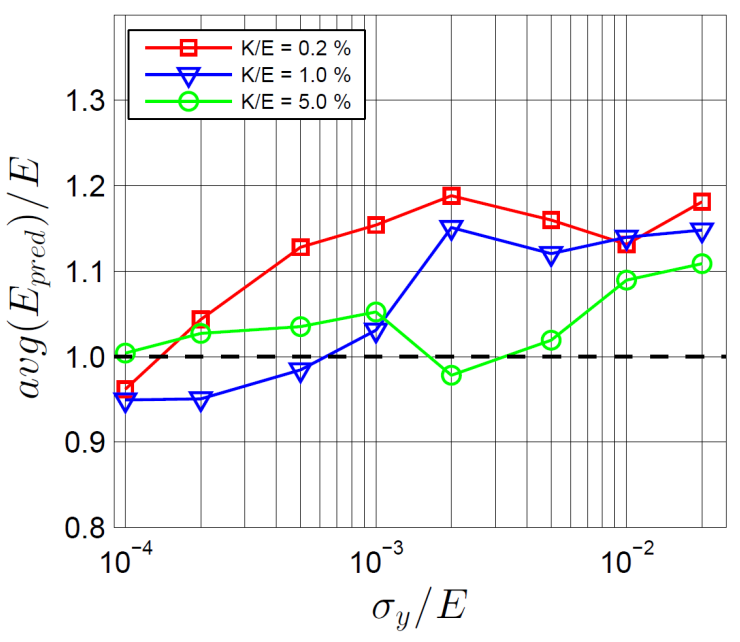

(b)

Figure 9: Results for power-law fitting of the equivalent indenter profile over the "contact" domain. (a) Comparison between the power-law exponents derived from fitting either the equivalent indenter profile or the load-penetration curve during the unloading stage. (b) Mean value of the Young's modulus.

Before leaving this section, a few considerations on the choice of the domain over which $\bar{f}_{e q}(\bar{x})$ is approximated need to be made as well. When the "contact" domain is replaced by the "extended" domain visualized in figure 4, the number of terms considered in equation (21) has to be increased, in order to follow the more complex shape of the residual impression beyond the point of contact at maximum load. However, it was observed that the accuracy of the Young's modulus calculation does not improve significantly compared to figure 7 , for all combinations of material parameters tested. This indicates that, concerning the description of the elastic contact between the real indenter and the surface containing a residual impression, the information contained in the shape of the $\bar{u}_{r}$ function beyond $\bar{a}_{m}$ does not probably play a major role.

\subsection{Definition of the inverse problem}

The previous section indicates that the equivalent indenter concept does indeed provide a way to describe the elastic unloading stage by means of analytical solutions which are formally valid only for indentation into a flat 
surface. The following paragraphs discuss how to use this result to extract information about the sample elastic constants from a real nano-indentation test.

Let us assume that the curves $P(t)$ and $\bar{h}(t)$ have been recorded during unloading, with $t$ denoting the time variable. The inverse problem consists in finding an equivalent indenter profile $\bar{f}_{e q}(\bar{x})$ and a value for the reduced Young's modulus $E^{*}$ such that relations (9) can be satisfied at any instant $t$ for a smooth monotonic function $\bar{a}(t)$.

On the basis of the previous findings, an expression of the type (27) is sufficient to approximate $\bar{f}_{e q}(\bar{x})$. Therefore, only 2 coefficients are needed to characterize the equivalent indenter profile. This means that the inverse problem could in principle be formulated as an optimization problem where suitable values for the 3 scalar unknowns $\bar{b}_{1}, \bar{b}_{3}$ and $E^{*}$ have to be determined, which provide values for $P(t)$ and $\bar{h}(t)$ according to Segedin's equations (11) - (13) which are as close as possible to those recorded experimentally. This formulation looks particularly attractive because any deviation of the real indenter from the ideal conical geometry could be naturally taken into account without the need for any area-function calibration.

The number of unknowns can be reduced by one if the dimensionless function

$$
\Phi(t)=\frac{W(t)}{P(t) h_{m}}
$$

is employed. It should be noted that experimental values for $\Phi$ are easily calculated, as the work $W(t)$ can be obtained from a nano-indentation curve via integration. By inserting (13) and (15) into the latter expression, one obtains

$$
\Phi\left(\bar{c}_{1}, \bar{c}_{3}, \bar{a}(t)\right)=\left(\sum_{n, s=1,3} \frac{n s}{(s+n)(n+1)} \bar{c}_{n} \bar{c}_{s} \bar{a}^{n+s+1}\right) \times\left(\sum_{n=1,3} \frac{n}{n+1} \bar{c}_{n} \bar{a}^{n+1}\right)^{-1}
$$

where the $\bar{c}_{n}$ coefficients are uniquely related to the $\bar{b}_{n}$ via equation (11). Furthermore, relation (12) can be inverted by means of common formulas for the roots of third-order polynomials. If the obtained expression $\bar{a}=r\left(\bar{h}, \bar{c}_{1}, \bar{c}_{3}\right)$ is inserted into (30), the original system reduces to the single equation

$$
\begin{aligned}
\Phi\left(\bar{c}_{1}, \bar{c}_{3}, \bar{h}(t)\right)= & \left(\sum_{n, s=1,3} \frac{n s}{(s+n)(n+1)} \bar{c}_{n} \bar{c}_{s} r\left(\bar{h}, \bar{c}_{1}, \bar{c}_{3}\right)^{n+s+1}\right) \times \\
& \left(\sum_{n=1,3} \frac{n}{n+1} \bar{c}_{n} r\left(\bar{h}, \bar{c}_{1}, \bar{c}_{3}\right)^{n+1}\right)^{-1}
\end{aligned}
$$


A standard optimization algorithm could then be used to determine values for $\bar{c}_{1}, \bar{c}_{3}$ which minimize the deviation with the experimental values of $\Phi$ over the range of $\bar{h}$ considered. After that, the reduced Young's modulus $E^{*}$ could be easily calculated from either (13) or (15) just replacing $\bar{a}$ with $r\left(\bar{h}, \bar{c}_{1}, \bar{c}_{3}\right)$.

\subsection{Non-uniqueness of the solution}

Unfortunately, the inverse problem previously formulated is not well-posed, in the sense that it does not admit a unique solution. In fact, let us assume that a solution $\left(\bar{c}_{1}, \bar{c}_{3}\right)$ has been found. It can be checked on common formulas for the roots of third-order polynomials that for any $k>0$ the following equation holds true

$$
r\left(\bar{h}, \bar{c}_{1}, \bar{c}_{3}\right)=k r\left(\bar{h}, k \bar{c}_{1}, k^{3} \bar{c}_{3}\right)
$$

By inserting this expression into equation (31) one can verify easily that

$$
\Phi\left(\bar{c}_{1}, \bar{c}_{3}, \bar{h}\right)=\Phi\left(k \bar{c}_{1}, k^{3} \bar{c}_{3}, \bar{h}\right)
$$

which proves that the pair $\left(k \bar{c}_{1}, k^{3} \bar{c}_{3}\right)$ is also solution to the inverse problem.

The non-uniqueness of the solution stems directly from the particular mathematical structure of the general equations (9), which implies that the same elastic load penetration curve can be generated by different indenter profiles in combination with different values of the reduced Young's modulus. Indeed, let us consider an indenter profile described by the function $f_{e q, 1}(x)$ and a value $E_{1}^{*}$ of the reduced Young's modulus. For a given value $\bar{a}_{1}$ of the contact radius, Sneddon's equations (9) deliver load and penetration as follows:

$$
\bar{h}=\int_{0}^{\bar{a}_{1}} \frac{f_{e q, 1}^{\prime}\left(h_{m} \bar{x}\right)}{\sqrt{1-\left(\bar{x} / \bar{a}_{1}\right)^{2}}} d \bar{x}, \quad P=2 E_{1}^{*} h_{m}^{2} \int_{0}^{\bar{a}_{1}} \frac{\bar{x}^{2} f_{e q, 1}^{\prime}\left(h_{m} \bar{x}\right)}{\sqrt{\bar{a}_{1}^{2}-\bar{x}^{2}}} d \bar{x}
$$

Now, let us introduce the quantities

$$
f_{e q, k}(x)=f_{e q, 1}(k x), \quad E_{k}^{*}=k E_{1}^{*}, \quad \bar{a}_{k}=\bar{a}_{1} / k
$$

with $k>0$.

By inverting the $2^{\text {nd }}$ and $3^{\text {rd }}$ of the relations (35) and inserting them into (34) one obtains

$$
\bar{h}=\int_{0}^{k \bar{a}_{k}} \frac{f_{e q, 1}^{\prime}\left(h_{m} \bar{x}\right)}{\sqrt{1-\bar{x}^{2} /\left(k \bar{a}_{k}\right)^{2}}} d \bar{x}, \quad P=2 \frac{E_{k}^{*}}{k} h_{m}^{2} \int_{0}^{k \bar{a}_{k}} \frac{\bar{x}^{2} f_{e q, 1}^{\prime}\left(h_{m} \bar{x}\right)}{\sqrt{k^{2} \bar{a}_{k}^{2}-\bar{x}^{2}}} d \bar{x}
$$

By noticing that

$$
f_{e q, 1}^{\prime}(x)=\frac{d f_{e q, 1}(x)}{d x}=\frac{d f_{e q, k}(x / k)}{d x}=\frac{d f_{e q, k}}{d(x / k)} \frac{d(x / k)}{d x}=\frac{1}{k} f_{e q, k}^{\prime}(x / k)
$$

one may rewrite (36) as 


$$
\bar{h}=\int_{0}^{k \bar{a}_{k}} \frac{f_{e q, k}^{\prime}\left(h_{m} \bar{x} / k\right)}{k \sqrt{1-\bar{x}^{2} /\left(k \bar{a}_{k}\right)^{2}}} d \bar{x}, \quad P=2 \frac{E_{k}^{*}}{k} h_{m}^{2} \int_{0}^{k \bar{a}_{k}} \frac{\bar{x}^{2} f_{e q, k}^{\prime}\left(h_{m} \bar{x} / k\right)}{k \sqrt{k^{2} \bar{a}_{k}^{2}-\bar{x}^{2}}} d \bar{x}
$$

Finally, by making the dummy variable substitution $\bar{x} / k=\bar{z}$ in the integrals appearing in the last formulas one obtains

$$
\bar{h}=\int_{0}^{\bar{a}_{k}} \frac{f_{e q, k}^{\prime}\left(h_{m} \bar{z}\right)}{\sqrt{1-\left(\bar{z} / \bar{a}_{k}\right)^{2}}} d \bar{z}, \quad P=2 E_{k}^{*} h_{m}^{2} \int_{0}^{\bar{a}_{k}} \frac{\bar{z}^{2} f_{e q, k}^{\prime}\left(h_{m} \bar{z}\right)}{\sqrt{\bar{a}_{k}^{2}-z^{2}}} d \bar{z}
$$

which show that the quantities $f_{e q, k}(x)$ and $E_{k}^{*}$ defined in (35) produce a load penetration curve identical to that associated with the indenter profile $f_{e q, 1}(x)$ and the reduced Young's modulus $E_{1}^{*}$. From a physical point of view, this means that if the indenter profile is made shallower (resp. steeper) by a scaling transformation quantified by the parameter $k$, the elastic force-penetration relation does not change if the reduced Young's modulus is increased (resp. decreased) correspondingly by the same scaling factor. Interestingly, the contact depth remains unaffected by this scaling transformation:

$$
h_{c, k}=f_{e q, k}\left(h_{m} \bar{a}_{k}\right)=f_{e q, 1}\left(k h_{m} \bar{a}_{k}\right)=f_{e q, 1}\left(h_{m} \bar{a}_{1}\right)=h_{c, 1}
$$

for any $k>0$.

One possibility to remove the non-uniqueness affecting the inverse problem identified by equation (31) is to introduce additional assumptions, which somehow pose limitations on the shape of the equivalent indenter. This could be achieved by prescribing the value of either $\bar{c}_{1}$ or $\bar{c}_{3}$ by means of a scalar condition of the type

$$
g\left(\bar{c}_{n}\right)=0, \quad n=1 \vee 3
$$

In principle, the function $g$ should be universal, i.e. independent of the material parameters. However, it seems more realistic to be able to construct one such function which depends on one or more experimentally accessible quantities which are related to the material properties. On the other hand, if the chosen $g$ turned out to be dependent on more than one parameter, the overall idea of using an analytical solution combined with the equivalent indenter concept would break down, as Dao et al. (Dao et al., 2001) showed that the reduced Young's modulus itself can be expressed as a function of two indentation parameters:

$$
E^{*}=\frac{P_{m}}{h_{m}^{2}} \Pi_{3}\left(\frac{P_{m}^{\prime} h_{m}}{P_{m}}, \bar{h}_{p}\right)
$$

where the subscript " $\mathrm{m}$ " denotes evaluation of the physical quantity at maximum load. Fortunately, it seems to be actually possible to find $g$ functions which depend on less than two parameters and, at the same time, allow solving the inverse problem to a sufficient level of accuracy in most practical cases. An example of this is given in the next section. 


\subsection{The Oliver-Pharr method}

In the following, it will be shown that the classic Oliver-Pharr method can be considered as a particular case of inverse problem consistent with the equivalent indenter concept as discussed in section 4.4, where very specific assumptions are made for the constraint (41). Remarkably, it turns out that these assumptions are intrinsically embedded in the apparent inconsistencies of the method which were pointed out in connection with expressions (1) and (3).

To begin with, it has to be noted that the latter of these relations can be rewritten in dimensionless form as

$$
\bar{a}=\bar{h}_{c} \tan (\alpha)
$$

due to the fact that $\bar{A}=\pi \bar{a}^{2}$. It is also useful to re-cast equation (1) in the following form

$$
\bar{h}_{c, O P}=\bar{h}+\bar{h}_{p}-\epsilon \frac{P}{P^{\prime}}
$$

We emphasize that now the symbol $\bar{h}$ appearing in the last expression denotes only the elastic part of the penetration, i.e. the difference between the total penetration recorded and the plastic residual penetration $\bar{h}_{p}$, both normalized by the total maximum penetration $h_{m}$. Hence, $\bar{h}+\bar{h}_{p}$ corresponds to the total penetration.

The first step to unveil the underlying assumptions of the method is to note that the factor $P / P^{\prime}$ appearing at the right-hand-side of equation (44) was derived in (Oliver and Pharr, 1992) from the original Sneddon's solution for a conical indenter, equation (18). By differentiating that expression, one can easily see that $P / P^{\prime}=\bar{h} / 2$. This result holds also for indenters with power-law profiles, as can be easily checked by taking the derivative of equation (16). By inserting it into (44) one obtains

$$
\bar{h}_{c, O P}=\bar{h}_{p}+(1-\epsilon / 2) \bar{h}
$$

As mentioned in the introduction, in the Oliver-Pharr method this formula is used only at maximum load. Consequently, it is possible to use the identity $\bar{h}+\bar{h}_{p}=1$ to rewrite it as

$$
\bar{h}_{c, O P}=\left(\frac{\bar{h}_{p}}{1-\bar{h}_{p}}+\frac{2-\epsilon}{2}\right) \bar{h}
$$

Finally, equations (43) and (46) can be combined together to obtain

$$
\bar{h}=\cot (\alpha)\left(\frac{\bar{h}_{p}}{1-\bar{h}_{p}}+\frac{2-\epsilon}{2}\right)^{-1} \bar{a}
$$

The last expression represents a linear relation between contact radius and elastic penetration. By comparing it with equation (12), it is immediately realized that the constant multiplying $\bar{a}$ at the right-hand-side corresponds to the $\bar{c}_{1}$ coefficient for an equivalent indenter with linear profile, whose slope is delivered by equation (17) as 


$$
\bar{b}_{O P}=\frac{2}{\pi} \bar{c}_{1}=\frac{2 \cot (\alpha)}{\pi}\left(\frac{\bar{h}_{p}}{1-\bar{h}_{p}}+\frac{2-\epsilon}{2}\right)^{-1}
$$

and whose semi-apical angle $\alpha_{O P}$ turns out to be

$$
\alpha_{O P}=\pi / 2-\operatorname{atan}\left(\bar{b}_{O P}\right)
$$

A few crucial observations can be made at this point. First of all, the Oliver-Pharr method can be considered as entirely based on the equivalent indenter concept, without any inconsistencies. Secondly, such equivalent indenter is assumed to be conical a priori. Thirdly, the associated semi-apical angle turns out to be given by equations (48)-(49). Consequently, it can be stated that the method is based on two constraints of the type (41): one forces $\bar{c}_{3}$ to zero and the other one, corresponding to equation (48), prescribes the value of $\bar{c}_{1}$ according to the recorded value of $\bar{h}_{p}$.

Concerning this last aspect, figure 10-a shows the dependence of $\alpha_{O P}$ on $\bar{h}_{p}$. Two different values for the constant $\epsilon$ are considered: 0.72 , which is typical of a conical indenter, and 0.75 , which relates to a paraboloid but is often used in practice as it seems to provide better results (Oliver and Pharr, 2004). It can be noted that when the material is perfectly elastic, i.e. $\bar{h}_{p}=0, \alpha_{O P}$ reduces to $\alpha=70.3^{0}$, the semi-apical angle of the real indenter. This happens exactly for $\epsilon=0.72$, whereas a small deviation is seen for $\epsilon=0.75$. Conversely, when the material exhibits extensive plastic deformation and $\bar{h}_{p}$ tends to one, $\alpha_{O P}$ approaches 90 degrees, independently of the $\epsilon$ value. This is sensible, as during loading the material conforms perfectly to the real indenter shape, which is retained during subsequent unloading due to negligible elastic recovery. A reason for the big success of the Oliver-Pharr method could be this concealed convenient choice of the equivalent indenter profile, which explains why the underlying elastic equations describe the unloading stage relatively well even in the presence of a non-negligible residual impression on the sample.

The present analysis is also useful to re-examine the predictive capabilities of the Oliver-Pharr method under a different perspective. As extensively discussed in the literature (Bolshakov and Pharr, 1998)(Hay et al., 1999)(Poon et al., 2008a) errors above $10 \%$ are likely when $\bar{h}_{p}$ grows beyond 0.7 , due to extensive plastic flow with associated pile-up, and these are usually larger for materials showing little amount of work-hardening. This seems to be the case also for the present findings, reported in figure 10-b. The visible strong influence of the hardening parameter indicates that, for a given value of $\bar{h}_{p}$, multiple values of the equivalent indenter angle $\alpha_{O P}$ would be required to match the theoretical value of Young's modulus. As a consequence, to further improve the accuracy of the method, equation (48), should be modified somehow to include a dependency on some other experimentally accessible quantities related to work-hardening. Nevertheless, this would make the method less 
attractive compared to other procedures suggested in the literature, for the reason explained in the last paragraph of section 4.5.

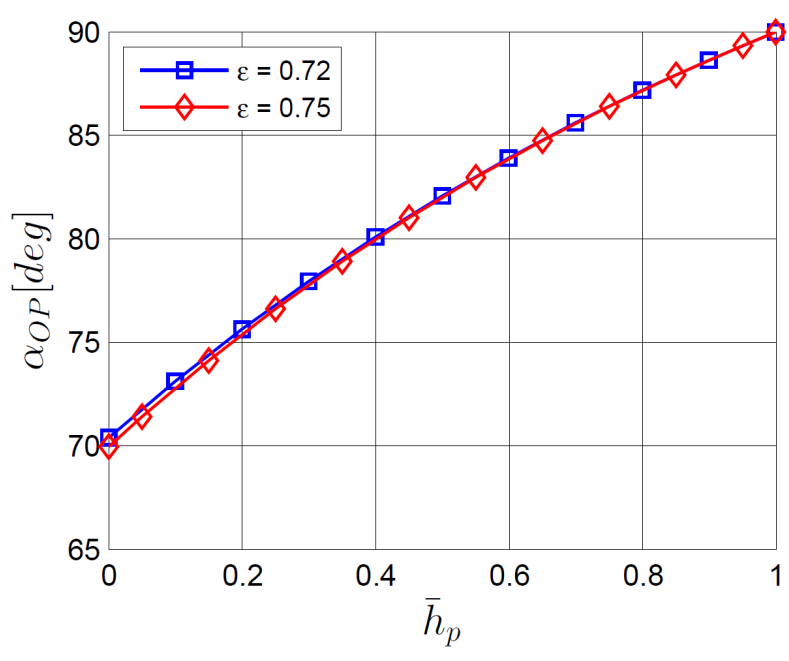

(a)

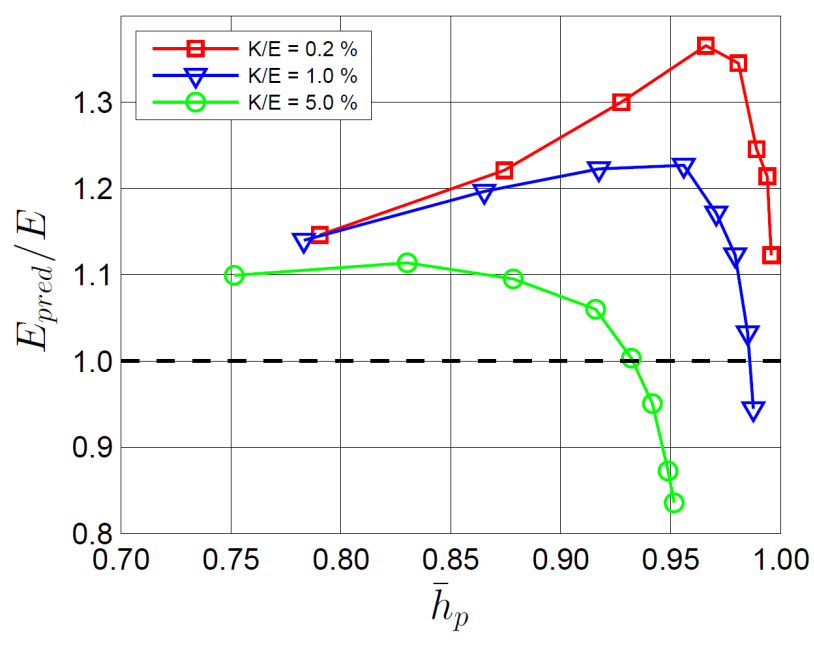

(b)

Figure 10: The Oliver-Pharr method. (a) Semi-apical angle of the implicitly assumed equivalent conical indenter as a function of the ratio between residual and maximum penetration. (b) Young's modulus predictions using the data of the present simulations.

\section{Conclusions}

The main findings of the present paper can be summarized as follows:

- The shape of the axisymmetric equivalent indenter can be considered as a material property and can be used to describe, within engineering accuracy, the nano-indentation unloading stage by means of Sneddon's elastic solution which is formally valid for indentation into a flat surface only.

- A consistent method of extracting the elastic moduli from nano-indentation data would imply solving an optimization problem where the unknowns are both the reduced Young's modulus and the shape of the equivalent indenter. This problem is ill-posed and additional assumptions are necessary to make its solution unique.

- One such assumption is hidden in the apparent inconsistencies of the Oliver-Pharr method, which may be seen as based on an equivalent conical indenter whose semi-apical angle depends on the ratio between residual and total penetration. Specifically, this angle tends to 90 degrees when the material exhibits extensive inelastic deformation, whereas it reduces to the one characteristic of the real indenter for a perfectly elastic material. 
- Altogether, these results offer a new physical explanation for why the elastic equations of the OliverPharr method, which are formally valid for contact with a perfectly flat surface, allow for a quite accurate calculation of the Young's modulus even in the presence of a residual impression on the sample during unloading.

\section{Acknowledgements}

This research was supported by the Department of Mechanical Engineering, Technical University of Denmark, and by the Strategic Research Center 'REWIND-Knowledge based engineering for improved reliability of critical wind turbine components', Danish Research Council for Strategic Research, grant no. 10-093966.

\section{References}

Ashby, M., Shercliff, H., Cebon, D., 2010. Materials : engineering, science, processing and design. Elsevier Butterworth-Heinemann.

Barenblatt, G.I., 1996. Scaling, Self-similarity, and Intermediate Asymptotics. Cambridge University Press, Cambridge.

Bolshakov, A., Oliver, W.C., Pharr, G.M., 1995. An explanation for the shape of nanoindentation unloading curves based on finite element simulation. Mater. Res. Soc. Symp. Proc. 356, 675-680.

Bolshakov, A., Pharr, G.M., 1998. Influences of pileup on the measurement of mechanical properties by load and depth sensing indentation techniques. J. Mater. Res. 13, 1049-1058.

Boussinesq, J., 1885. Applications des potentiels a l'etude de equilibre et du mouvement des solides elastiques, Gauthier-Villars. Paris.

Cheng, Y.-T., Cheng, C.-M., 1998. Relationships between hardness, elastic modulus, and the work of indentation. Appl. Phys. Lett. 73, 614. doi:10.1063/1.121873

Cheng, Y.-T., Cheng, C.-M., 1999. Scaling relationships in conical indentation of elastic-perfectly plastic solids. Int. J. Solids Struct. 36, 1231-1243. doi:10.1016/S0020-7683(97)00349-1

Cheng, Y.-T., Cheng, C.-M., 2004. Scaling, dimensional analysis, and indentation measurements. Mater. Sci. Eng. R Reports 44, 91-149. doi:10.1016/j.mser.2004.05.001

Cheng, Y.-T., Li, Z., Cheng, C.-M., 2002. Scaling relationships for indentation measurements. Philos. Mag. A 82, 1821-1829. doi:10.1080/01418610210135043

Dao, M., Chollacoop, N., Van Vliet, K.J., Venkatesh, T.A., Suresh, S., 2001. Computational modeling of the forward and reverse problems in instrumented sharp indentation. Acta Mater. 49, 3899-3918. doi:10.1016/S1359-6454(01)00295-6

Dassault Systèmes Simulia Corp., 2013. Abaqus 6.13, Abaqus Analysis User's Guide section 12.2.7.

Doerner, M.F., Nix, W.D., 1986. A method for interpreting the data from depth-sensing indentation instruments. J. Mater. Res. 1, 601-609. doi:10.1557/JMR.1986.0601

Fischer-Cripps, A.C., 2006. Critical review of analysis and interpretation of nanoindentation test data. Surf. Coatings Technol. 200, 4153-4165. doi:10.1016/j.surfcoat.2005.03.018

Fischer-Cripps, A.C., 2011. Nanoindentation. Springer.

Gibson, R.F., 2014. A review of recent research on nanoindentation of polymer composites and their constituents. Compos. Sci. Technol. 105, 51-65. doi:10.1016/j.compscitech.2014.09.016

Gong, J., Miao, H., Peng, Z., 2004. Analysis of the nanoindentation data measured with a Berkovich indenter for brittle materials: effect of the residual contact stress. Acta Mater. 52, 785-793. 
doi:10.1016/j.actamat.2003.10.013

Greaves, G.N., Greer, A.L., Lakes, R.S., Rouxel, T., 2011. Poisson's ratio and modern materials. Nat. Mater. 10, 823-837. doi:10.1038/nmat3134

Harding, J.W., Sneddon, I.N., 1945. The elastic stresses produced by the indentation of the plane surface of a semi-infinite elastic solid by a rigid punch. Proc. Camb. Philol. Soc. 41, 16-26.

Hay, J., Crawford, B., 2011. Measuring substrate-independent modulus of thin films. J. Mater. Res. 26, 727-738. doi:10.1557/jmr.2011.8

Hay, J.C., Bolshakov, A., Pharr, G.M., 1999. A critical examination of the fundamental relations used in the analysis of nanoindentation data. J. Mater. Res. 14, 2296-2305.

Hay, J., Agee, P., Herbert, E., 2010. Continuous stiffness measurement during instrumented indentation testing. Exp. Tech. 34, 86-94. doi:10.1111/j.1747-1567.2010.00618.x

Larsson, P.-L., Giannakopoulos, A.E., SÖderlund, E., Rowcliffe, D.J., Vestergaard, R., 1996. Analysis of Berkovich indentation. Int. J. Solids Struct. 33, 221-248. doi:10.1016/0020-7683(95)00033-7

Li, Z., Cheng, Y.-T., Yang, H.T., Chandrasekar, S., 2002. On two indentation hardness definitions. Surf. Coatings Technol. 154, 124-130. doi:10.1016/S0257-8972(02)00021-X

Love, A.E.H., 1939. Bousinnesq's problem for a rigid cone. Q. J. Math. os-10, 161-175. doi:10.1093/qmath/os10.1.161

Mata, M., Alcala, J., 2003. Mechanical property evaluation through sharp indentations in elastoplastic and fully plastic contact regimes. J. Mater. Res. 18, 1705-1709.

McKee, C.T., Last, J.A., Russell, P., Murphy, C.J., 2011. Indentation Versus Tensile Measurements of Young's Modulus for Soft Biological Tissues. Tissue Eng. Part B Rev. 17, 155-164. doi:10.1089/ten.teb.2010.0520

Ni, W., Cheng, Y.-T., 2005. Modeling conical indentation in homogeneous materials and in hard films on soft substrates. J. Mater. Res. 20, 521-528. doi:10.1557/JMR.2005.0071

Nix, W.D., 1989. Mechanical properties of thin films. Metall. Trans. A 20, 2217-2245. doi:10.1007/BF02666659

Oliver, W.C., Pharr, G.M., 1992. An improved technique for determining hardness and elastic modulus using load and displacement sensing indentation experiments. J. Mater. Res. 7, 1564-1580.

Oliver, W.C., Pharr, G.M., 2004. Measurement of hardness and elastic modulus by instrumented indentation: Advances in understanding and refinements to methodology. J. Mater. Res. 19, 3-20.

Pharr, G.M., Bolshakov, A., 2002. Understanding nanoindentation unloading curves. J. Mater. Res. 17, 2660 2671.

Pharr, G.M., Oliver, W.C., Brotzen, F.R., 1992. On the generality of the relationship among contact stiffness, contact area, and elastic modulus during indentation. J. Mater. Res. 7, 613-617.

Pharr, G.M., Strader, J.H., Oliver, W.C., 2009. Critical issues in making small-depth mechanical property measurements by nanoindentation with continuous stiffness measurement. J. Mater. Res. 24, 653-666. doi:10.1557/jmr.2009.0096

Poon, B., Rittel, D., Ravichandran, G., 2008a. An analysis of nanoindentation in elasto-plastic solids. Int. J. Solids Struct. 45, 6399-6415. doi:10.1016/j.ijsolstr.2008.08.016

Poon, B., Rittel, D., Ravichandran, G., 2008b. An analysis of nanoindentation in linearly elastic solids. Int. J. Solids Struct. 45, 6018-6033.

Saha, R., Nix, W.D., 2002. Effects of the substrate on the determination of thin film mechanical properties by nanoindentation. Acta Mater. 50, 23-38. doi:10.1016/S1359-6454(01)00328-7

Sakharova, N.A., Fernandes, J.V., Antunes, J.M., Oliveira, M.C., 2009. Comparison between Berkovich, Vickers and conical indentation tests: A three-dimensional numerical simulation study. Int. J. Solids Struct. 46, 1095-1104. doi:10.1016/j.ijsolstr.2008.10.032

Segedin, C.M., 1957. The relation between load and penetration for a spherical punch. Mathematika 4, 156. doi:10.1112/S0025579300001236

Sneddon, 1948. Boussinesq's problem for a rigid cone. Proc. Camb. Philol. Soc. 44, 492-507.

Sneddon, I.N., 1965. The relation between load and penetration in the axisymmetric boussinesq problem for a 
punch of arbitrary profile. Int. J. Eng. Sci. 3, 47-57. doi:10.1016/0020-7225(65)90019-4

Troyon, M., Huang, L., 2005. Critical Examination of the Two-slope Method in Nanoindentation. J. Mater. Res. 20, 2194-2198. doi:10.1557/JMR.2005.0272

Troyon, M., Huang, L., 2006. Comparison of different analysis methods in nanoindentation and influence on the correction factor for contact area. Surf. Coatings Technol. 201, 1613-1619. doi:10.1016/j.surfcoat.2006.02.033

Tyulyukovskiy, E., Huber, N., 2006. Identification of viscoplastic material parameters from spherical indentation data: Part I. Neural networks. J. Mater. Res. 21, 664-676. doi:10.1557/jmr.2006.0076

Tyulyukovskiy, E., Huber, N., 2007. Neural networks for tip correction of spherical indentation curves from bulk metals and thin metal films. J. Mech. Phys. Solids 55, 391-418. doi:10.1016/j.jmps.2006.07.003

Wang, J.S., Zheng, X.J., Zheng, H., Zhu, Z., Song, S.T., 2010. Evaluation of the substrate effect on indentation behavior of film/substrate system. Appl. Surf. Sci. 256, 5998-6002. doi:10.1016/j.apsusc.2010.03.108

Wang, L., Rokhlin, S.I., 2005. Universal scaling functions for continuous stiffness nanoindentation with sharp indenters. Int. J. Solids Struct. 42, 3807-3832. doi:10.1016/j.ijsolstr.2004.11.012

Woirgard, J., Dargenton, J.-C., Tromas, C., Audurier, V., 1998a. A new technology for nanohardness measurements: principle and applications. Surf. Coatings Technol. 100-101, 103-109. doi:10.1016/S02578972(97)00597-5

Woirgard, J., Dargenton, J.C., 1997. An alternative method for penetration depth determination in nanoindentation measurements. J. Mater. Res. 12, 2455-2458.

Woirgard, J., Tromas, C., Girard, J.C., Audurier, V., 1998b. Study of the mechanical properties of ceramic materials by the nanoindentation technique. J. Eur. Ceram. Soc. 18, 2297-2305. doi:10.1016/S09552219(98)00083-1

Xu, H., Pharr, G.M., 2006. An improved relation for the effective elastic compliance of a film/substrate system during indentation by a flat cylindrical punch. Scr. Mater. 55, 315-318. doi:10.1016/j.scriptamat.2006.04.037 